\title{
Person Verification using Fusion of Palm Geometry and Palm Print
}

\author{
Suhas S. Chate* \\ Student, Dept. of E\& T C \\ College of Engineering, \\ Pune-411005, India.
}

\author{
Pratik R. Ubale \\ Student, Dept. of E\& T C \\ College of Engineering, \\ Pune-411005, India.
}

\author{
M.A. Joshi ,Ph.D \\ Professor, Dept. of E\& T C \\ College of Engineering, \\ Pune-411005, India.
}

\begin{abstract}
This paper proposes a biometric person verification system based using hand images. This paper attempts to improve the performance of palm print-based verification system by integrating hand geometry features at different levels of fusion. In this work geometrical features such as finger lengths and finger widths are used. For extraction of the palm print features Gabor filter based approach is utilized. For matching, the newly acquired biometric samples are compared with those stored in the system database, at the enrolment stage. Unlike other bimodal biometric systems, the users do not have to undergo the inconvenience of using two different sensors since the palm print and hand geometry features can be acquired from the same image, using single capturing device at the same time. The capturing device has a resolution of $640 \times 480$ pixels. Our experimental results on the image dataset from 300 users confirm the utility of combining hand geometry features with those from palm prints using a simple image acquisition setup. It also illustrates the performance of different fusion methods such as decision level, score level. FAR of $0.28 \%$ and FRR of $2.0 \%$ is achieved for the database using score level fusion of palm geometry and palm print.
\end{abstract}

\section{General Terms}

Image Processing, Pattern Recognition, Biometrics, Security, Personal Verification.

\section{Keywords}

Palm geometry, Palm print, Multi-Biometrics, Fusion.

\section{INTRODUCTION}

Biometrics is the automated method of recognizing a person based on a physiological or behavioral characteristic. Biometric authentication is gaining popularity as a more trustable alternative to password based security systems as it is relatively hard to be forgotten, stolen, or guessed [1]. Palm based person verification systems, mainly palm/finger geometry based verification systems are widely accepted amongst other biometric traits. This can be seen from their increasing commercial deployments.

*The Corresponding Author is Currently DGFS-PhD Scholar
at Bhabha Atomic Research Center, Mumbai, India. \# The Corresponding Author is Currently Programmer Analyst with Cognizant Technology Solutions , Pune, India.
Though the systems have achieved significant commercial success, several issues are to be considered to have more userfriendly systems. Major problems include, inconvenience caused by the constrained imaging set up, especially to elderly and people suffering from limited dexterity. It is also seen that geometrical features (hand/finger geometry or silhouette) extracted from the palm have limited discriminatory information and are not known to be highly distinctive [1] Thus many researchers are moving towards fusion of multiple biometrics to achieve more sophistication in the present systems.

Instead of using multiple biometrics such as palm and face, fingerprint and palm; a single biometric entity can be analysed with multiple methods for feature extraction. Human palm is one of such biometric modality. The palm presents rich geometrical features as well as rich texture features in terms of principle lines present on it [2]. Essentially, hand verification methods available in the literature can be classified in to three categories based upon the nature of image acquisition [3].

- Constrained and contact based: These systems make use of pegs or pins to constrain the position of hand. Most commercial systems and early research systems fall under this category.

- Unconstrained and contact based: Hand images are captured in an unconstrained manner. This required the users to place their hand on flat surface or a digital scanner.

- Unconstrained and contact-free: This approach does away with the need for any pegs or platform during hand image acquisition. This mode of image acquisition is believed to be more user-friendly and have recently received increased attention from biometric researchers.

\section{PRIOR WORK}

Most hand-based biometric schemes in the literature are based on measuring the hand silhouette as a distinctive personal attribute for an authentication task. As the work began it is peg based systems that shared most of the research interest. The guiding pegs provide consistent measuring positions; they cause some problems as well including 1) the pegs can deform the shape of a hand [3] and 2) The users must be well trained to use the system. There are two main approaches for geometrical features extraction; those based on measure the finger lengths and widths at various positions, palm size, etc. 
and another based on represent the global hand shape [4]. Both approaches use the finger tip points and the finger valley points as the land marks for image alignment. The palm texture can be also used as biometric trait for personal verification. Recently, perhaps due to hygiene consideration, contact-free hand biometric systems have been proposed. The two main issues to be dealt with in a contact-free system are hand segmentation and the projective distortions associated with the absence of the contact plane [4].

Saroj Kumar Panigrahy et. Al developed an efficient palm print image recognition system. The authors have suggested a rotational- as well as translational-invariant scheme by which the above problem can be overcome while pre-processing the image before the feature extraction of the palm print. The developed system is based on optical scanner and had greater computational complexity [5]. [4] Presented a contactless, bi spectral hand based biometric authentication system using geometric and palm features. Hand images were acquired using two commercial webcams with $1200 \mathrm{X} 1600$ pixel resolution, which are referred to as 'IR' and 'Visible' webcams. The IR webcam has been modified by exchanging IR filter with a visible filter lens and reducing the gain and exposure time to improve the hand contour extraction. The hand is illuminated using 24 infrared LEDs and 4 White light LEDs. Images acquired from the IR webcam were binarized and then normalized widths from the index to little finger were used as features.

A Least square SVM is employed for verification. The approach for extracting region of interest is complex. Training SVM is a difficult and time consuming task.[6] proposed simple contactless acquisition device. It does not have any auxiliary instruments of any form. After image acquisition, geometry features are extracted based on key points, which are selected by Sliding Window Filtering Algorithm. Experiments show that EER (Equal Error Rate) of the system reaches $2.16 \%$ and $2.40 \%$ in two different kinds of data sets. [7] Represented Multimodal Biometrics Recognition System Combined Palm Print and Palm Geometry Features. The system has a single sensor for image capturing. The Intensity Normalization technique for pre-processing is complex. Weighted score level fusion is proposed. Pegs are placed for proper positioning of hand. FAR (False Acceptance Rate) and FRR (False Rejection Rate) of the system reaches $0.71 \%$ and $2.67 \%$.

\section{IMPLEMENTATION}

\subsection{Image Acquisition module and Database preparation}

Figure 1 shows the block diagram for the proposed system. The system consists of four major blocks: Image acquisition module, image pre-processing block, feature extraction and verification. The entire system diagram is briefly described as follows. First, the palm print and hand geometry image acquisition module uses a digital camera to capture the hand images, and then the pre processing module employs image processing algorithms to demarcate the region of interest (R.O.I) from an input image. This module performs noise reduction and smoothening of boundary. Next, the feature extraction module extracts the features of hand geometry and palm print. Finally, recognition module employs a minimum distance classifier according to city block distance metric to recognize the hand pattern by comparing the feature vector with the enrolled data in the database. A special image acquisition setup is developed for database generation. The acquisition setup consists of a black box with a slit at a side and a window in the front as shown in the Figure 2. The black box eliminates the effect of the ambient lighting during image capture. It also helps to solve the problem of background elimination [4]. The captured images are of size $640 \times 480$ pixels.. The database consists of images from 300 individuals. 8 images for each person were captured over a period of 2 months. Out of the 8 images 4 are used for training purpose and remaining 4 are tested against the designed system.

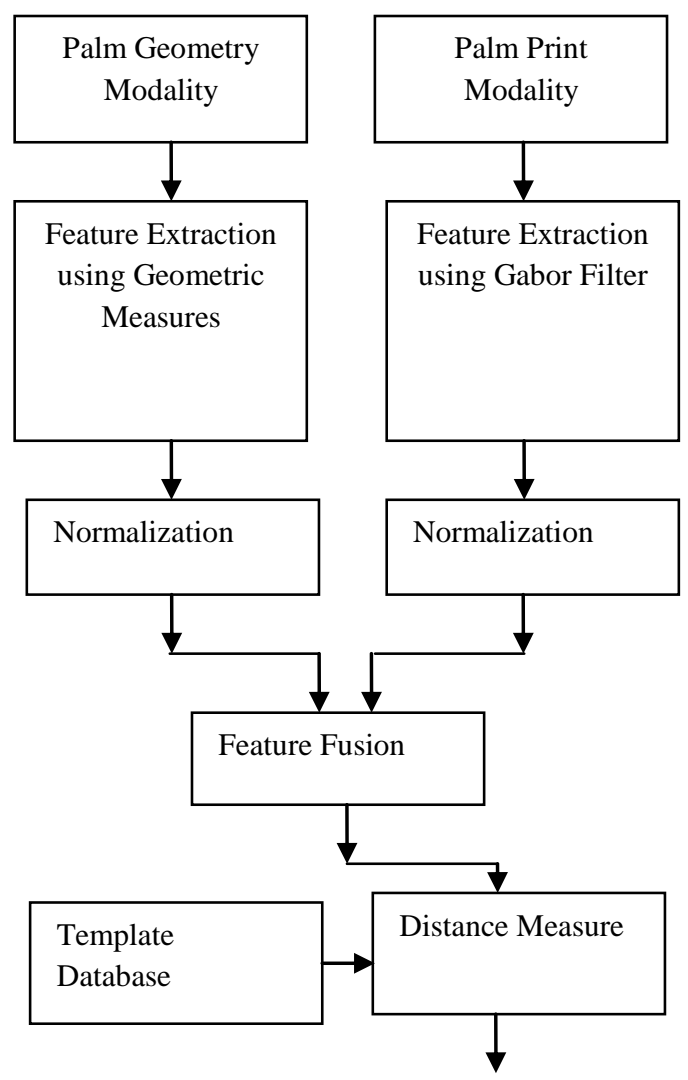

Fig 1: System Architecture

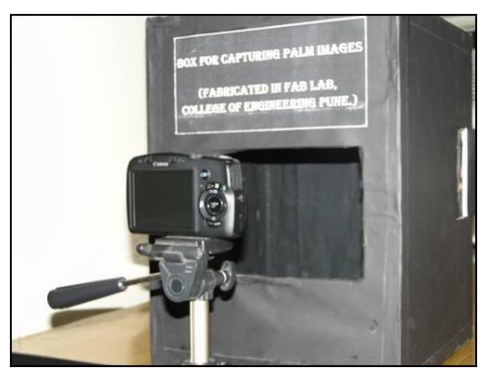

Fig 2: Image Acquisition Setup

\subsection{Feature Extraction}

\subsubsection{Pre-processing}

Captured image is converted to gray scale. Image is filtered using Gaussian filter in order to remove any noise which may cause problems while thresholding the image. The proposed 
method uses Gaussian filter $\mathrm{G}(\mathrm{x}, \mathrm{y})$ on the original image, $\mathrm{I}(\mathrm{x}$, $y)$ to obtain a blur version of the image $\mathrm{M}(\mathrm{x}, \mathrm{y})$.

$$
M(x, y)=M(x, y) * I(x, y)
$$

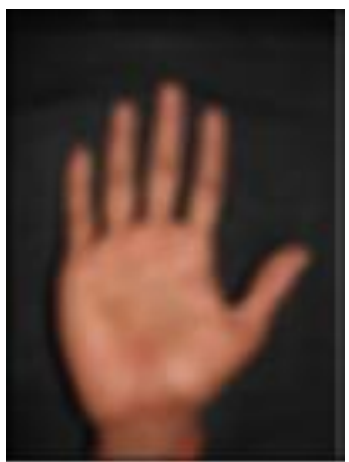

Fig 3: Image after Applying Gaussian Filter

This filtered image is converted to binary image using a global threshold $\mathrm{T}$. For determining the threshold the Iso data thresholding method proposed by El-Zaart [10] is used. The steps are as follows.

- Calculate the grayscale histogram of the image.

- Obtain the mean from the histogram and assign it as the threshold To.

- Separate the histogram into two groups R1 and R2 by To.

- Obtain the two mean values $\mathrm{m} 1$ and $\mathrm{m} 2$ from $\mathrm{R} 1$ and R2.

- Calculate a new threshold $\mathrm{T}$ is obtained by $\mathrm{T}=(\mathrm{m} 1$ $+\mathrm{m} 2) / 2$.

- Assign $\mathrm{T}=\mathrm{To}$, Repeat above steps till achieving a convergent $\mathrm{T}$.

- The converged threshold $\mathrm{T}$ is then employed to binarize the grayscale image as follows. Let $\mathrm{M}(\mathrm{x}, \mathrm{y})$ be the input image and $\mathrm{N}(\mathrm{x}, \mathrm{y})$ be thresholded image.

$$
\begin{aligned}
& N(x, y)=0 \text { if } M(x, y)<T \\
& N(x, y)=1 \text { if } M(x, y) \geq T
\end{aligned}
$$

Since the binarized result might generate some notch edges on the contour of the palm, the "erosion" and "dilation" operations from morphology is utilized to reduce this effect. Suzuki [11] developed a contour extraction algorithm using border following. The same method is used here to extract the hand contour from binarized image.
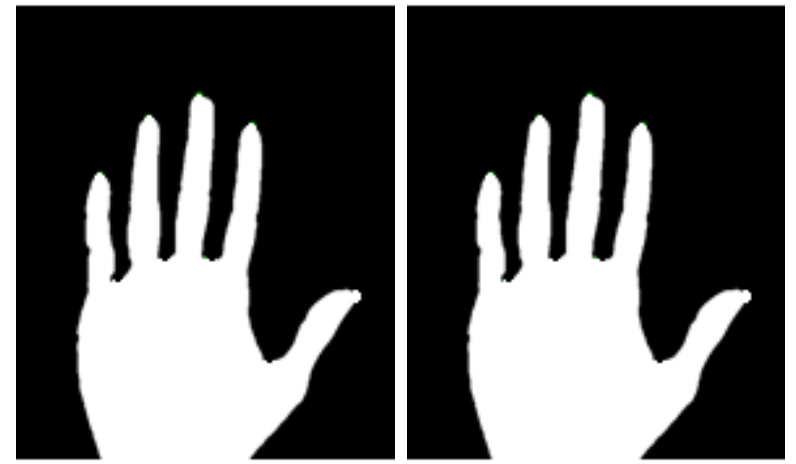

Fig 4 a): Binary Image b) Extracted Contour

\subsubsection{Geometrical Features and Region of Interest (ROI) Extraction}

The hand contour plays very important role in extracting the tip and valley points of the palm. This step has much importance for the further processing and algorithm because the output image from this step has all reference points i.e. fingertips and valley points, which are also useful to extract the Region of Interest (ROI) of palm. To locate the peak and valley points on the hand boundary, the basic steps are as follows.

- Find the lower left most point of the palm in the binarized image.

- Find the lower right most point of the palm in the binarized image.

- $\quad$ Find the midpoint of the above two points this mid point is used as reference point for tip and valley point detection.

- Find the Euclidean distance of all contour pixels with respect to the reference point and plot these all distance with respect to index of all points.

The maxima in the plot represent the tip points and the minima represent the valley points of the palm [12]. Hand geometry is considered to achieve medium security, but with several advantages compared to other techniques:

- Medium cost as it only needs a platform and medium resolution reader or camera.

- It uses low-computational cost algorithm, which leads to fast results.

- Low template size, which reduces the storage needs.
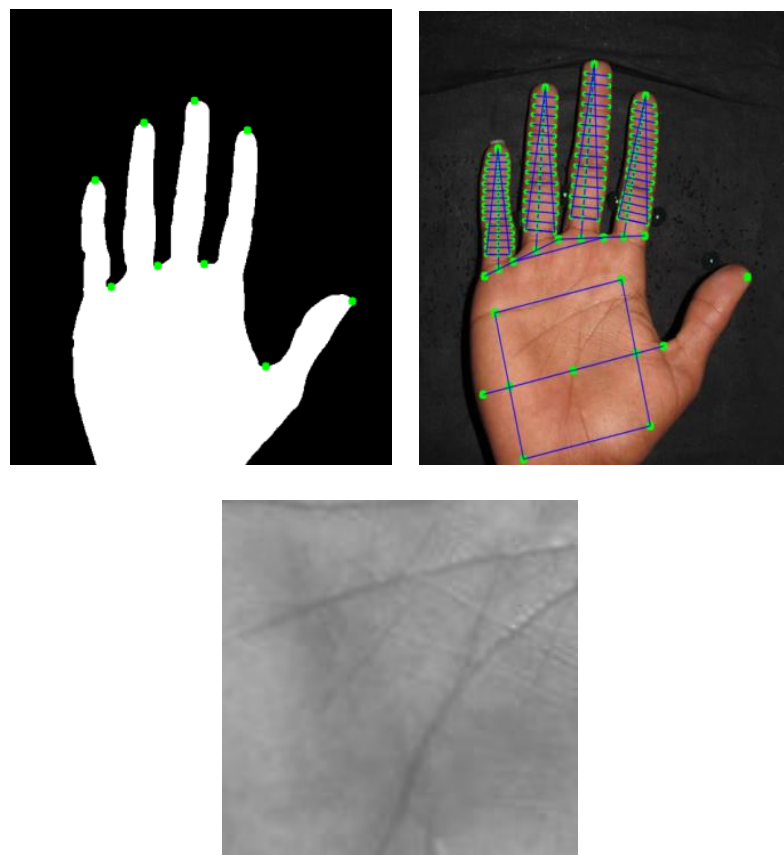

Fig 5: a) Location of tip and valley points b) Geometrical Features c) Extracted ROI 
Steps involved in extraction of geometrical features are

- Locate the base lines for the finger.

- Locate middle points of all the base lines. The middle point between two points $\left(\mathrm{X}_{1}, \mathrm{Y}_{1}\right)$ and $\left(\mathrm{X}_{2}\right.$, $\mathrm{Y}_{2}$ )

- $\quad$ Finger lengths are calculated by Euclidian Distance between respective fingertip points and middle points of the respective base lines.

- Perpendiculars are drawn on the finger lengths at constant spacing to calculate the finger width. Euclidian Distance between two points $\left(\mathrm{X}_{1}, \mathrm{Y}_{1}\right)$ and $\left(\mathrm{X}_{2}, \mathrm{Y}_{2}\right)$ is given by,

$$
E D=\sqrt{\left(X_{1}-X_{2}\right)^{2}+\left(Y_{1}-Y_{2}\right)^{2}}
$$

Total 72 geometrical features (4 finger lengths, 15 widths per finger and sides of triangle joining finger tip point and baseline end points of finger.) are taken in to consideration. Figure $5 \mathrm{~b}$ ) shows the 72 geometrical features. Based on the location of tip and valley points a region of interest (ROI) consisting of line features of palm is extracted as shown in figure 3.The extracted ROI is of size $128 \mathrm{X} 128$ pixels.

\subsubsection{Palm print Feature Extraction}

The palm print features are extracted using Gabor filter. The palm print has fascinating texture information.

Therefore, it is attractive to search representation methods which can capture the local crucial information in a palm print. There have been many techniques suggested in the literature for extracting unique and invariant features from the palm print image. These techniques employ either texture- or appearance-based features. Gabor filter is used for feature extraction. General form of a 2D Gabor filter is defined by

$$
\begin{aligned}
& h\left(x, y, \theta_{k}, f, \sigma_{x}, \sigma_{y}\right)=\exp \left[-\frac{1}{2}\left(\frac{x_{\theta_{k}}{ }^{2}}{\sigma_{x}{ }^{2}}+\frac{y_{\theta_{k}}{ }^{2}}{\sigma_{y}{ }^{2}}\right)\right] \times \\
& \exp \left(12 \pi f x_{\theta_{k}}\right)
\end{aligned}
$$

Wherex $_{\theta_{\mathrm{k}}}=\mathrm{x} \cos \theta_{\mathrm{k}}+\mathrm{y} \sin \theta_{\mathrm{k}}$ andy $_{\theta_{\mathrm{k}}}=\mathrm{y} \cos \theta_{\mathrm{k}}-\mathrm{x} \sin \theta_{\mathrm{k}}$, $\mathrm{f}$ is the frequency of the sinusoidal plane wave, $\theta_{\mathrm{k}}$ is the orientation of the Gabor filter, and $\sigma_{\mathrm{x}}$ and $\sigma_{\mathrm{y}}$ are the standard deviations of the Gaussian envelope along the $\mathrm{x}$ and $\mathrm{y}$ axes, respectively. Figure 6 shows response of a Gabor filter.

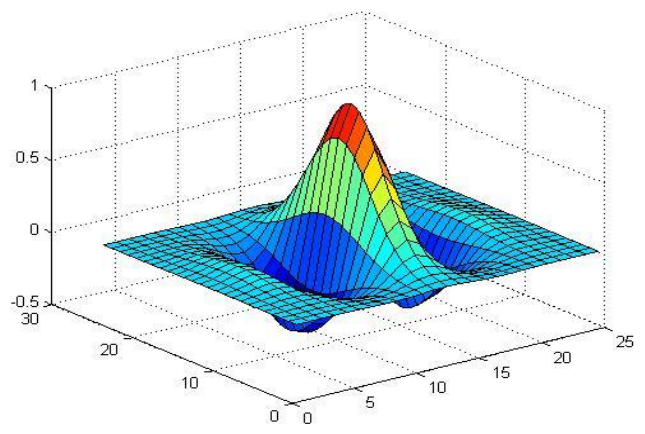

Figure 6: Response of Gabor filter
Gabor filters are employed because they have frequency-selective and orientation-selective properties [13]. These properties allow the filter to be tuned to give maximal response to patterns at a specific orientation and frequency in the palm print image.

Therefore, a properly tuned Gabor filter can be used to effectively preserve the pattern structures while reducing noise. The ROI image is processed with the help of four directional Gabor filter bank having orientation in 0,45 , 90,135 degrees respectively. The four processed images are transformed into feature vectors by computing standard deviation of $32 \times 32$ pixel block of the processed image. The following figures show the phase response of the Gabor filter.
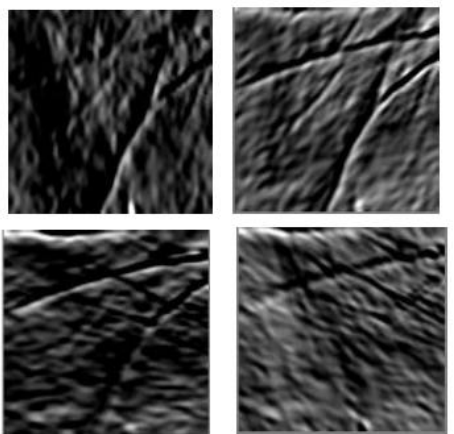

Fig 7: Phase Response of Gabor filter in four orientations

\subsection{Fusion of Palm Geometry and Palm Print}

In order to increase the accuracy of the system fusion of palm geometry and palm print is done. These approaches are discussed in [11].

\subsubsection{Decision level fusion}

It is the simplest method of fusion in which decision of person verification is taken based on thresholds obtained by both the modalities. So if the test features satisfy both the criteria then only the test feature is accepted to be from genuine person.

\subsubsection{Score level fusion}

In score level fusion the output scores from the matcher of both the modalities are first normalized and then combined either by concatenation or addition or sometimes subtraction to get resultant score. This combined score is then used for verification.

\subsection{Feature Normalization}

In this work four techniques for score normalization have been employed. They are briefly explained below.

\subsubsection{Min-Max normalization}

The simplest normalization technique is the Min-max normalization. Min-max normalization is best suited for the case where the bounds (maximum and minimum values) of the scores produced by a matcher are known. In this case, one can easily shift the minimum and maximum scores/features to 0 and1, respectively. However, even if the matching scores are not bounded, one can estimate the minimum and 
maximum values for a set of matching scores/features and then apply the min-max normalization. Given a set of matching scores $\{\mathrm{sk}\}, \mathrm{k}=1,2, \ldots, \mathrm{n}$, the normalized scores/features are given by

$$
\frac{(s-\min (s))}{(\max (s)-\min (s))}
$$

When the minimum and maximum values are estimated from the given set of matching scores, this method is not robust (i.e., the method is highly sensitive to outliers in the data used for estimation). Min-max normalization retains the original distribution of scores except for a scaling factor and transforms all the scores/features into a common range $[0,1]$.

\subsubsection{Z-score normalization}

The most commonly used score normalization technique is the $\mathrm{z}$-score that is calculated using the arithmetic mean and standard deviation of the given data. This scheme can be expected to perform well if prior knowledge about the average score and the score variations of the matcher is available. The normalized scores are given by

$$
\frac{(s-\text { 回 })}{\sigma}
$$

Where $\mu$ the arithmetic mean and $\sigma$ is the standard deviation of the given data. If the input scores/features are not Gaussian distributed, z-score normalization does not retain the input distribution at the output. This is due to the fact that mean and standard deviation are the optimal location and scale parameters only for a Gaussian distribution. For an arbitrary distribution, mean and standard deviation are reasonable estimates of location and scale, respectively, but are not optimal.

\subsubsection{MAD normalization}

The median and median absolute deviations (MAD) are insensitive to outliers and the points in the extreme tails of the distribution. Hence, a normalization scheme using median and MAD would be robust and is given by

$$
\frac{(s-\text { median })}{M A D}
$$

Where $\mathrm{MAD}=$ median $(\mid \mathrm{s}-$ median $\mid)$. However, the median and the MAD estimators have a low efficiency compared to the mean and the standard deviation estimators, i.e., when the score distribution is not Gaussian, median and MAD are poor estimates of the location and scale parameters. Therefore, this normalization technique does not retain the input distribution and does not transform the scores/features into a common numerical range.

\subsubsection{Tanh normalization}

The tanh-estimators introduced by Hampel et al. are robust and highly efficient. The normalization is given by

$$
\frac{1}{2}\left[\tanh \left(0.01 \frac{(s-0)}{\sigma}\right)\right]
$$

$\mu$ and $\sigma$ are mean and standard deviation estimates, respectively.

\subsection{Feature Matching}

The palm geometry feature template is of 288 bytes consisting of 72 float values. The palm print feature template is of 256 bytes having 64 float values. Testing feature template is compared with stored average feature template using distance metrics. City-block distance is used for palm geometry features. Canberra distance is used for palm print features.

\subsubsection{City-block/Manhattan Distance}

Hermann Minkowski considered the city-block distance. Absolute difference at each dimension of feature vector is known as city-block or Manhattan distance.

$$
d(x, y)=\sum_{j=1}^{N}\left|x_{j}-y_{i}\right|
$$

\subsubsection{Canberra distance}

The Canberra metric is similar to the Manhattan distance. The distinction is that the absolute difference between the variables of the two objects is divided by the sum of the absolute variable values prior to summing.

$$
\begin{gathered}
d(x, y)=\sum_{j=1}^{N} \frac{\left|x_{j}-y_{i}\right|}{\left|x_{j}\right|+\left|y_{i}\right|} \\
\text { Where, } x_{j}=\text { Testing feature vector } \\
y_{i}=\text { Trained feature vector } \\
N=\text { Total number of features }
\end{gathered}
$$

\section{RESULTS AND DISCUSSIONS}

Out of eight acquired images for each user four are used as for training and other four are used for testing purpose. First individual results for palm geometry and palm print are compared. The two modalities are then fused at different levels. Total minimum error rate (TMER) is used as a metric for system performance evaluation [2].When using TMER as a performance metric the sum of FAR and FRR is taken into consideration. The threshold where the sum is minimum is used as decision metric. Table 1 describes False Acceptance Rate (FAR) and False Rejection Rate (FRR) for different features. Figure 8 represents FAR and FRR curves for both the modalities.

Table 1. FAR and FRR for different types of feature

\begin{tabular}{|c|c|c|c|}
\hline $\begin{array}{c}\text { Types of } \\
\text { features }\end{array}$ & $\begin{array}{c}\text { Types Of } \\
\text { Distances }\end{array}$ & FAR (\%) & FRR (\%) \\
\hline $\begin{array}{c}72 \text { palm } \\
\text { geometry } \\
\text { features }\end{array}$ & $\begin{array}{c}\text { City } \\
\text { block }\end{array}$ & 0.97 & 7.08 \\
\hline $\begin{array}{c}\text { Gabor } \\
\text { features of } \\
\text { palm print }\end{array}$ & Canberra & 0.68 & 3.16 \\
\hline
\end{tabular}




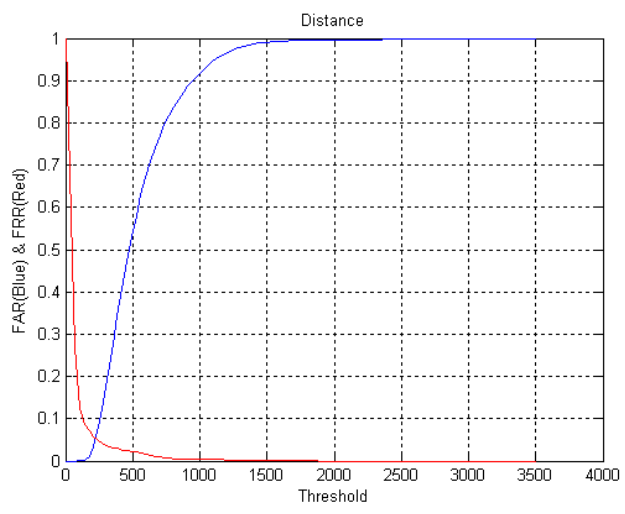

Fig. 8 a) FAR and FRR for 72 palm geometry features

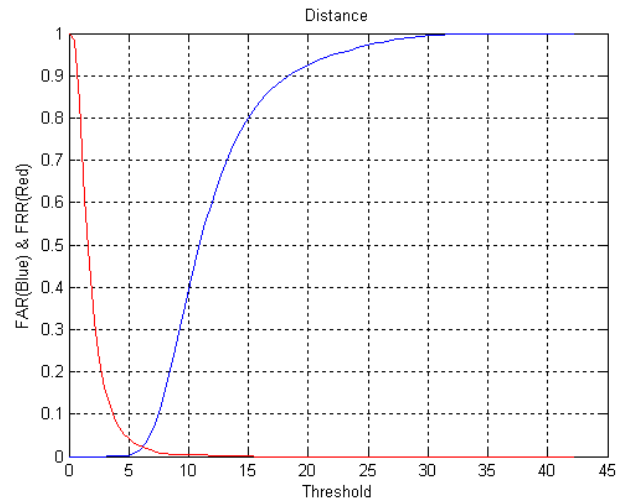

Fig. 8 b) FAR and FRR for Gabor features of palm print feature

Table 2 describes False Acceptance Rate (FAR) and False Rejection Rate (FRR) for decision level fusion.

Table 2. FAR and FRR for decision level fusion

\begin{tabular}{|c|c|c|c|}
\hline $\begin{array}{c}\text { Types of } \\
\text { features }\end{array}$ & $\begin{array}{c}\text { Types Of } \\
\text { Distances }\end{array}$ & FAR (\%) & FRR (\%) \\
\hline $\begin{array}{c}72 \text { palm } \\
\text { geometry } \\
\text { features+ } \\
\begin{array}{c}\text { Gabor } \\
\text { features of } \\
\text { palm print }\end{array}\end{array}$ & $\begin{array}{c}\text { City } \\
\text { block and } \\
\text { Canberra }\end{array}$ & 0.022 & 8.92 \\
\hline
\end{tabular}

Table 3 describes False Acceptance Rate (FAR) and False Rejection Rate (FRR) for score level fusion. Here the 72 geometric features and Gabor features of palm print are combined at score level. For score level fusion four normalization techniques are used viz. Min max normalization, Medial Axis Deviation, Z-score normalization and tanh normalization. This combined score is then used to verify the persons. Table 3 describes False Acceptance Rate (FAR) and False Rejection Rate (FRR) for score level fusion.
Table 3. FAR and FRR for score level fusion

\begin{tabular}{|c|c|c|}
\hline $\begin{array}{c}\text { Type } \\
\text { Normalization }\end{array}$ & FAR $(\boldsymbol{\%})$ & FRR (\%) \\
\hline Min-Max & 1 & 7.8 \\
\hline $\begin{array}{c}\text { Medial Axis } \\
\text { Deviation }\end{array}$ & 1.2 & 7 \\
\hline Z-Score & 0.59 & 7.33 \\
\hline Tanh & 0.28 & 2.0 \\
\hline
\end{tabular}

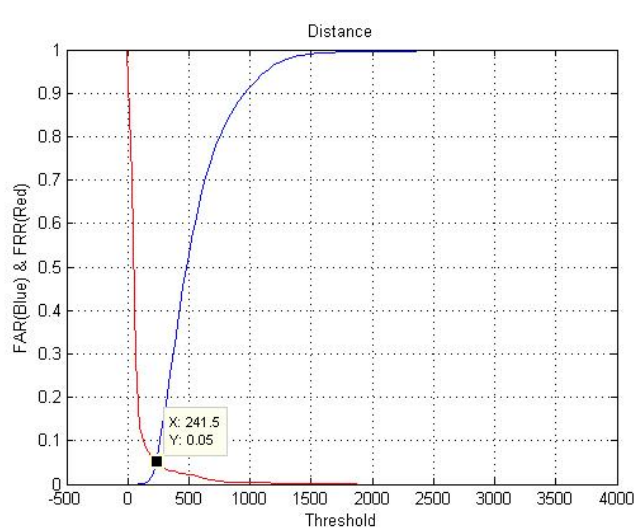

Fig. 9 a) FAR and FRR for score level fusion using ZScore normalization

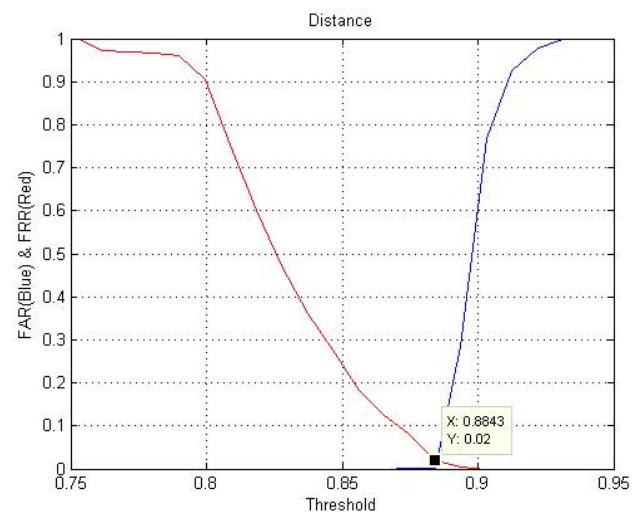

Fig. 9 b) FAR and FRR for score level fusion using Tanh normalization

\section{CONCLUSIONS}

In this work an user verification system based on palm geometry and palm print has been developed. In this work different technique of fusion of the two traits viz. Decision level fusion, score level fusions are compared. The algorithms used for extracting region of interest of palm print are less complex as against [4, 7]. Features extracted from individual modalities gave better results as compared to $[2,6]$. Finally the features obtained from palm geometry and palm print are fused at decision level, score level to increase recognition accuracy of the system. It is observed that Decision level fusion works best making FAR as low as $0.02 \%$. But it increases FRR to $9 \%$ as two level decisions is 
made. Score level fusion works best giving FAR of $0.28 \%$ and FRR of $2.0 \%$ for a database of 300 persons. Score level fusion with good results has medium computational complexity. Decision level fusion gave FAR of $0.026 \%$ which is excellent figure. But as two level decision is made the scheme has a FRR of 9\%. But it is quite fast and simple. Score level normalization scheme performed well using tanh normalization giving FAR of $0.28 \%$ and FRR of $2 \%$.

\section{ACKNOWLEDGMENTS}

This work has been produced with the support of Rajiv Gandhi Science and Technology Commission, Government of Maharashtra, India.

\section{REFERENCES}

[1] Anil K. Jain, Arun Ross and Salil Prabhakar, "An Introduction to Biometric Recognition", IEEE Transactions on Circuits and Systems for Video Technology, Vol. 14, No. 1, January 2004.

[2] Ajay Kumar, David C. M. Wong, Helen C. Shen1, Anil K. Jain, Personal Verification using Palmprint and Hand Geometry Biometric IEEE Transactions on Image Processing, VOL. 15, NO. 8, AUGUST 2006

[3] Vivek Kanhangad, Ajay Kumar and David Zhang, "Contactless and Pose Invariant Biometric Identification Using Hand Surface," IEEE Transactions on Image Processing, Vol. 20, No. 5, May 2011, pp.1415-1424

[4] A.Wong and P. Shi, "Peg-free hand geometry recognition using hierarchical geometry in and shape matching," Proc. IAPR Workshop on Machine Vision Applications, Nara, Japan, Dec. 2002, pp. 281-284 [Online]. Available: http://www.ee.ust.hk/ eeship/Papers/MVA02.pdf.

[5] Miguel A. Ferrer, Francisco Vargas, Aythami Morales, BiSpectral Contactless hand based biometric system. 2nd National Conference on Tele-communication, 2011, page $1-6$.
[6] Saroj Kumar Panigrahy, Debasish Jena and Sanjay Kumar Jena, "An Efficient Palm print Recognition System", Proceedings of National Conference on Soft Computing, SynSoft-08, 19th-20th Jan 2008, SIET, Dhenkanal, 2008.

[7] Cui Xin, Xiangqian Wu, Zhao Qiushi, Tang Youbao, A Contactless Hand Shape Identification System, 3rd International Conference on Advanced Computer Control (1CACC 2011)

[8] Yanuar Adhinagara, Tjokorda Agung B. W., Retno Novi Dayawati ,Implementation of Multimodal Biometrics Recognition System Combined Palm Print And Palm Geometry Features, 2011 International Conference on Electrical Engineering and Informatics 17-19 July 2011, Bandung, Indonesia

[9] Aythami Morales, Miguel A. Ferrer, Ajay Kumar," Improved Palm print Authentication using Contactless Imaging" Proceedings of Fourth International conference on Biometrics: theory applications and systems, 2010.

[10] A. El-Zaart, "Images thresholding using Isodata technique with gamma distribution," Pattern Recognition and Image Analysis, vol. 20, no. 1, pp. 29-41, 2010.

[11] Suzuki, S. and Abe, K., Topological Structural Analysis of Digitized Binary Images by Border Following. CVGIP 301 , pp 32-46

[12] Zhong Qu, Zheng-yong Wang, Research on preprocessing of Palmprint image based on adaptive threshold and Euclidean distance, Natural Computation ICNC 2010 sixth international conference, page 42384242 .

[13] David Zhang, Wai-Kin Kong, Jane You, and Michael Wong, Online Palm print Identification, IEEE Transaction on Pattern Analysis and Machine Intelligence, Vol. 25, No. 9, September 2003

[14] Anil Jain, Karthik Nandakumar, Arun Ross, Score normalization in multimodal biometric systems, Elsevier Pattern Recognition 38 (2005) 2270 - 2285 